\title{
Evolutionary multi-objective route and fleet assignment optimisation for regular and non-regular flights
}

\author{
Keiki Takadama* \\ The University of Electro-Communications, \\ 1-5-1, Chofugaoka, Chofu, Tokyo 182-8585, Japan \\ Email: keiki@inf.uec.ac.jp \\ *Corresponding author
}

\section{Takahiro Jinba}

Mizuho Information and Research Institute, 2-3 Kanda-Nishikicho, Chiyoda-ku, Tokyo 101-8443, Japan

Email: takahiro412.c@gmail.com

\section{Tomohiro Harada}

Ritsumeikan University,

1-1-1 Noji-higashi, Kusatsu, Shiga 525-8577, Japan

Email: harada@ci.ritsumei.ac.jp

\section{Hiroyuki Sato}

The University of Electro-Communications, 1-5-1, Chofugaoka, Chofu, Tokyo 182-8585, Japan Email: sato@hc.uec.ac.jp

\begin{abstract}
To optimise the flight schedule that consists of: 1) the regular flight operated on the same day and time through one year; 2) the non-regular flight operated on the different day and time according to month, this paper proposes the new multi-objective fleet assignment method that considers both the regular and non-regular flights. To investigate the effectiveness of our method, this paper applies it to Japanese domestic airport network optimisation for two months, on- and off-peak months, using a real-world data. The intensive simulation have revealed that the following implications: 1) our method can evolve a flight network that can be applied into the on- and off-peak month; 2) our method can find a flight network that has a well-balanced profit between on- and off-peak months; 3) in peak month, our method can find a flight network that has higher profit.
\end{abstract}

Keywords: multi-objective optimisation; regular and non-regular flights optimisation; evolutionary algorithm; fleet assignment problem; FAP. 
Reference to this paper should be made as follows: Takadama, K., Jinba, T., Harada, T. and Sato, H. (2016) 'Evolutionary multi-objective route and fleet assignment optimisation for regular and non-regular flights', Int. J. Automation and Logistics, Vol. 2, Nos. 1/2, pp.122-152.

Biographical notes: Keiki Takadama received his Doctor of Engineering degree from the University of Tokyo, Japan in 1998. He joined Advanced Telecommunications Research Institute (ATR) International from 1998 to 2002 as a Visiting Researcher and worked at Tokyo Institute of Technology from 2002 to 2006 as a Lecturer. He moved to The University of Electro-Communications as an Associate Professor in 2006 and is currently a Professor since 2011.

Takahiro Jinba received his BE degree in 2013 and ME degree in 2015, both from the University of Electro-Communications, Japan, respectively. He joined Mizuho Information and Research Institute since 2015.

Tomohiro Harada received his ME degree in 2012 and earned Doctor of Engineering degree in 2015, both from the University of ElectroCommunications, Japan, respectively. He moved to Ritsumeikan University as an Assistant Professor in since 2015.

Hiroyuki Sato received his MS degree in 2005 and earned Doctor of Engineering degree in 2009, both from Shinshu University, Japan, respectively. $\mathrm{He}$ moved to the University of Electro-Communications as an Assistant Professor in since 2009.

This paper is a revised and expanded version of a paper entitled 'Multi objective optimization for route planning and fleet assignment in regular and non-regular flights' presented at the 18th Asia Pacific Symposium on Intelligent and Evolutionary Systems (IES 2014), Singapore, 10-12 November 2014.

\section{Introduction}

In order to generate the flight network for a different demand of passengers in each season, airline companies consider the regular and non-regular flights separately (Sato and Adachi, 2001). The regular flights are operated on the same day and time through one year, while the non-regular flights are operated on the different day and time according to operated month. Since the current rate of the non-regular flights in the whole flights is approximately $30 \%$ (Sato et al., 2001) and the number of the non-regular flights is increasing every year, the airline company should additionally cope with the non-regular flights. For these reasons, the optimisation for the regular and the non-regular flight network is required. However, the conventional methods cannot optimise both the regular and non-regular flight networks because they are designed for only optimising the flight network for one season (or single month) meaning that they cannot optimise it for several seasons (or multiple months). To understand this reason, let's focus on Table 1 which categorises the flight network optimisation. Historically, this research area starts to address either: 
1 the route planning problem (Clarke et al., 1997; Gopalan and Talluri, 1998)

2 fleet assignment problem (FAP) (Adachi et al., 2004; Gu et al., 1994; Hane et al., 1995) because of the difficulties of optimising both issues, and recently integrates them to conduct

3 the route planning and FAP (Barnhart et al., 1998; Gen et al., 2008; Sato and Adachi, 2001).

Table 1 Flight network optimisation

\begin{tabular}{lccc}
\hline & 1. Regular planning & 2. Fleet assignment & 1. + 2. \\
\hline $\begin{array}{l}\text { Regular flight } \\
\text { (single month) }\end{array}$ & $\begin{array}{c}\text { Gopalan and } \\
\text { Talluri (1998) and } \\
\text { Clarke et al. (1997) }\end{array}$ & $\begin{array}{c}\text { Adachi et al. (2004), } \\
\text { Hane et al. (1995) } \\
\text { and Gu et al. (1994) }\end{array}$ & $\begin{array}{c}\text { Gen et al. (2008), } \\
\text { Sato et al. (2001) } \\
\text { and Barnhart et al. } \\
(1998)\end{array}$ \\
$\begin{array}{l}\text { Regular + non-regular } \\
\begin{array}{l}\text { flight (multiple } \\
\text { months) }\end{array}\end{array}$ & & & $\begin{array}{c}\text { Proposed method } \\
\end{array}$ \\
\hline
\end{tabular}

As shown in Table 1, all methods can be applied for the regular flight (based on single month), but cannot be applied for the non-regular flight (based on multiple months). Note that the only research that addresses the non-regular flight optimises the crew pairing (but not optimise the flight network) (Sato et al., 2001).

To tackle this problem, this paper proposes the new route planning and fleet assignment method for the flight network optimisation that can assign aircrafts for both the regular and non-regular flights. This method is categorised in the right-bottom area as shown in Table 1. For this issue, our method focuses on the priority-based GA (PriGA) with connection network model (CNM) (Gen et al., 2008) which optimises the flight route and assignment of aircraft's type for the regular flights by considering passenger's demand. PriGA with CNM is based on the evolutionary computation as one of metaheuristics methods. We focus on it because the target problem is NP-hard [precisely, the FAP with more than two fleet types is NP-hard (Gu et al., 1994)], which means that the conventional mathematical programming methods are very hard to solve the target program from a practical viewpoint (in other words, evolutionary computation is one of potential candidates for NP-hard problem to find solutions within a practical time).

For this purpose, we extend PriGA with CNM by proposing:

1 the MIN-/MAX-based fleet assignment methods that assign the aircrafts to the flight network with the lower/higher passengers' demand in off/on peak months

2 the weight-based fleet assignment method which adjusts the number of the regular flights according to the weight values

3 the double self-adaptive fleet assignment method for both the MIN-/MAX-based and weight-based fleet assignment methods to determine an appropriate assignment (MIN or MAX) and evolve suitable weight values. 
To investigate the effectiveness of these methods, we conduct an experiment using a real-world data (i.e., the transportation cost of aircraft, the sales of airline, and the passenger's demand) and compare with the results of PriGA (the novel flight network optimisation for the regular flights) as a reference comparison.

This paper is organised as follows. Section 2 introduces the conventional route optimisation method, i.e., PriGA with CNM. Section 3 describes the mechanism of our proposed methods. Section 4 explains the domestic airport network problem. Section 5 applies our methods on the problem and their results are analysed. Finally, our conclusions and future works are presented in Section 6.

\section{Conventional route planning and feet assignment method}

\subsection{Precondition}

To conduct the route planning and fleet assignment, PriGA with CNM assumes the following preconditions:

1 a daily origin-destination (OD) table that indicates the passengers' demand of each flight route is given

2 the preparing cost of fleet is given depending on each departure airport

3 the number of aircrafts owned by an airline company is unlimited.

Table 2 Example of OD table

\begin{tabular}{lcc}
\hline $\mathrm{A}$ & $J$ & $B$ \\
\hline $\mathrm{B}$ & 0 & 30 \\
\hline
\end{tabular}

Regarding OD, Table 2 shows an example of OD table. In this table, the row indicates a departure airport, the column indicates a destination airport, and each cell shows the passengers' demand, i.e., the number of persons from the airport $i$ to $j$ (hereafter we call it as the OD value). For an example, 30 persons in Table 2 want to go to the airport B from A.

Regarding the cost, the cost of the flight schedule is defined as the summation of the transportation cost and the preparing cost of aircrafts for their maintenance.

\subsection{PriGA with CNM}

PriGA is an evolutionary route optimisation algorithm to optimise the several number of loop-type of routes. PriGA has been successfully applied to solve the vehicle routing problem (VRP) (Dantzig and Ramser, 1959), the ship routing problem (Inoue, 2012) and the FAP (Gen et al., 2008) focused on in this work. 


\subsubsection{Route representation and its regeneration}

In PriGA with CNM, each flight network is represented by a chromosome with an integer string as shown in Figure 1. This example has five airports (i.e., A, B, C, D, and E), and all airports are mutually connected. Each gene locus of the chromosome indicates an airway, the first row indicates the gene index numbers, the second row indicates the departure airports, the third row indicates the destination airports, and the last row indicates priority value of the gene in the chromosome. The gene length corresponds to the number of airways. Each priority value in the chromosome represents the priority of the airway, and a high value of chromosome indicates a high priority. Note that the same value in a chromosome is not prohibited in this gene representation. In Gen et al. (2008), the flight network represented by this gene structure is evolved by applying genetic operators such as crossover and mutation.

Figure 1 Route representation of PriGA with CNM (see online version for colours)

\begin{tabular}{|c|c|c|c|c|c|c|c|c|c|c|c|c|c|c|c|c|c|c|c|}
\hline Gene index & 1 & 2 & 3 & 4 & 5 & 6 & 7 & 8 & \multicolumn{3}{|c|}{\begin{tabular}{l|l|l|l|}
9 & 10 & 11 & 12
\end{tabular}} & \multicolumn{4}{|c|}{\begin{tabular}{|l|l|l|l}
13 & 14 & 15 & 16 \\
\end{tabular}} & \multicolumn{4}{|c|}{\begin{tabular}{|l|l|l|l|}
17 & 18 & 19 & 20 \\
\end{tabular}} \\
\hline Departure airport & \multicolumn{4}{|c|}{ A } & \multicolumn{4}{|c|}{ B } & \multicolumn{4}{|c|}{ C } & \multicolumn{3}{|c|}{ D } & \multicolumn{4}{|c|}{ E } \\
\hline Destination airport & B & C & D & E & A & c & D & E & A 1 & B & & 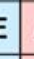 & A & 3 & $c \mid$ & & \begin{tabular}{l|l}
$A$ & B \\
\end{tabular} & c & D \\
\hline aromosome (priority) & 12 & 16 & & 10 & 19 & 17 & 9 & 18 & & 2 & 3 & 3 & 7 & 1 & 45 & & 1513 & 6 & 4 \\
\hline
\end{tabular}

The flight network generation procedure of PriGA with CNM is shown as follows:

1 The index $i$ having the highest priority is searched from the set of not selected indices. In Figure 1, for example, the gene 9 (which has 20 priority value) is selected.

2 The departure $i^{\text {th }}$ airport is set as $O_{i}$ of a route. In Figure 1, the airport $\mathrm{C}$ represented in 'departure airport' in gene 9 is set as $O_{l}$.

3 The destination $i^{\text {th }}$ airport is set as $D_{i}$, and the flight route from $O_{i}$ to $D_{i}$ is generated. In Figure 1, the airport A represented in 'destination airport' in gene 9 is set as $D_{l}$, and the flight route from the airport $\mathrm{C}$ to $\mathrm{A}$ is generated.

4 If the route does not return to the airport $O_{i}$, an index $i$ having the highest priority in $D i$ is selected from the set of not selected indices, and return to step (3). In the case of just after generating the flight route from the airport $\mathrm{C}$ to $\mathrm{A}$, the route does not return to the airport $\mathrm{C}$, the gene 2 (which has 16 priority value, i.e., the highest priority in the airport A) in Figure 1 is selected and return to step (3).

5 The steps (1) to (4) are repeated until all indices are selected. For example, after generating the flight route from C-A-C, the gene 5 (which has 19 priority value) is selected to continue to generate flight routes with the remaining gene indices.

\subsubsection{Fleet assignment}

After all flight routes are generated, the fleet type should be determined in the flight route to minimise the total costs while satisfying flight routing and constraints such as passengers' demand. This problem is called the FAP (Gen et al., 2008). Note that the FAP does not decide the time of the flight schedule but decides a flight route, the number of flights, and a fleet type assignment for each flight route. 
The FAP is formulised as follows, which is slightly modified from Abara (1989). Note that $i, j$, and $A$ indicate departure and destination airports and a set of airports, $f$ and $F$ indicate a fleet type and a set of fleet types, $C_{f}$ indicates a cost when flying with the fleet type $f, x_{i j k}$ is a decision variable (integer value) which indicates the number of flights from the airport $i$ to $j$ with the fleet type $f, X_{i}$ and $X_{j}$ indicate the maximum flights from the airport $i$ and to the airport $j$. The objective of this problem is to find appropriate $x_{i j k}$ for minimising the cost of flights as shown in equation (1) under the constraints of the cover shown in equation (2), balance shown in equation (3), and availability shown in equation (4). In detail, equation (2) indicates that the number of flights between arbitrary airports is 1 and over, meaning that there is a flight between arbitrary airports (i.e., the passengers' demand should be covered by all flights). Equation (3) indicates that the number of flights to the airport $l$ and that from the airport $l$ is the same under the same fleet type, meaning that the aircraft flow is balanced (not accumulated), i.e., the number of all types of aircrafts should be the same at the beginning and end of the day in all airports; finally, equation (4) indicates that the number of flights between arbitrary airports should not exceed the maximum flights from the airport $i$ and to the airport $j$, meaning that there is an availability of flights until $X_{i}$ and $X_{j}$ number of flights.

$$
\begin{array}{ll}
\text { Minimize } & \sum_{i \in A} \sum_{j \in A} \sum_{f \in F} C_{f} x_{i j k} \\
\text { Subject to } \quad \sum_{i \in A} \sum_{f \in F} x_{i j k} \geq 1 \quad \forall j \in A \\
\sum_{i \in A} x_{i l f}-\sum_{j \in A} x_{l j f}-0 \quad \forall l \in A, \quad f \in F \\
\sum_{j \in A} \sum_{f \in F} x_{i j k} \leq X_{i} \quad \forall i \in A, \quad \sum_{i \in A} \sum_{f \in F} x_{i j k} \leq X_{j} \quad \forall j \in A
\end{array}
$$

Figure 2 Flight route (A-B-C-A) (see online version for colours)

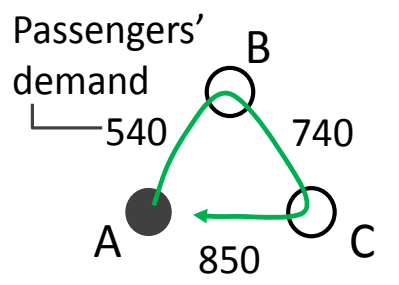

To understand the above equations [mainly equation (1)], this sub-section explains the fleet assignment method (Gen et al., 2008) by using an example of a flight route shown in Figure 2 [note that other equations (2), (3), and (4) are implicitly considered in the route generation described in Section 2.2.1]. In this figure, the nodes and the arrow indicate the airports and the flight route, respectively. This flight route includes three airways. This figure shows the passengers' demands between two airports for each airway. In this flight route, the aircraft departs from the airport A, and returns to the airport A via the airports $\mathrm{B}$ and $\mathrm{C}$. The procedure of the fleet assignment method is summarised as follows. 
1 The maximum value among the passengers' demands in all airways is determined as MaxFlow. In Figure 2, for example, the maximum value among the minimum passengers' demands (540, 740, and 850) is 850 and MaxFlow is set as 850 .

2 The type and number of aircrafts for each flight route are assigned according to MaxFlow. In detail, a fleet assignment that has the minimum cost is selected from all available combinations of aircraft under the condition of MaxFlow and some constraints (e.g., the number of aircraft). Assuming the large and small aircrafts, for example, the combinations of the fleet assignment that satisfy MaxFlow (850) are four patterns as shown in the right-side table of Figure 3. After calculating the costs of all combinations, a combination of aircrafts that has the minimum cost is selected. In this case, one large aircraft and two small aircrafts are assigned.

Figure 3 Fleet assignment according to the MaxFlow (see online version for colours)
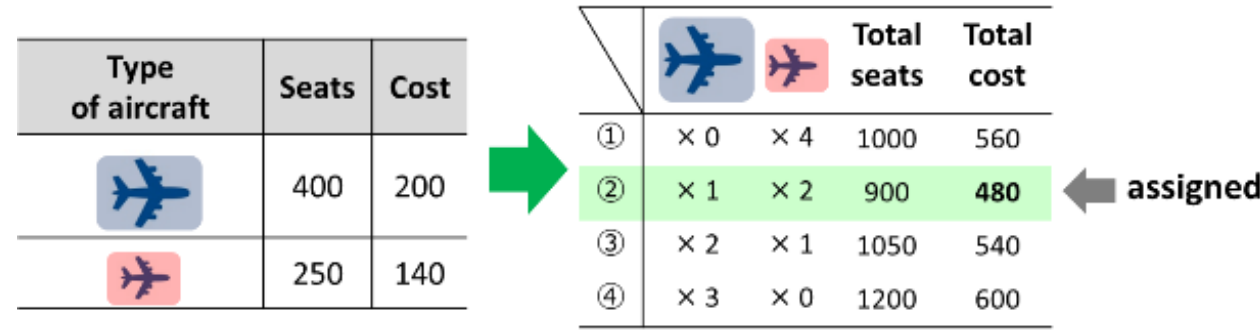

\subsubsection{Algorithm}

Figure 4 shows the architecture of PriGA with CNM. This example shows a flight network having three airports. In this case, the gene length becomes six. The flight network decoded from the gene has two routes [i.e., the clockwise route (green) and counter clockwise route (blue)]. The algorithm of PriGA with CNM is summarised as follows:

1 the initial population is randomly generated while avoiding the duplication of gene values in each individual

2 all individuals in the population are decoded to generate flight networks and their fitness are calculated (see in Section 5.2.2)

3 the parent individuals are selected according to the fitness

4 the offspring are generated by applying the crossover operator

5 the mutation operator is conducted with a predetermined mutation rate

6 the worse individuals having a low fitness in the population is replaced with the generated offspring

7 the steps (2)-(6) are repeated until the predetermined termination condition is satisfied. 
Figure 4 Architecture of PriGA with CNM (see online version for colours)

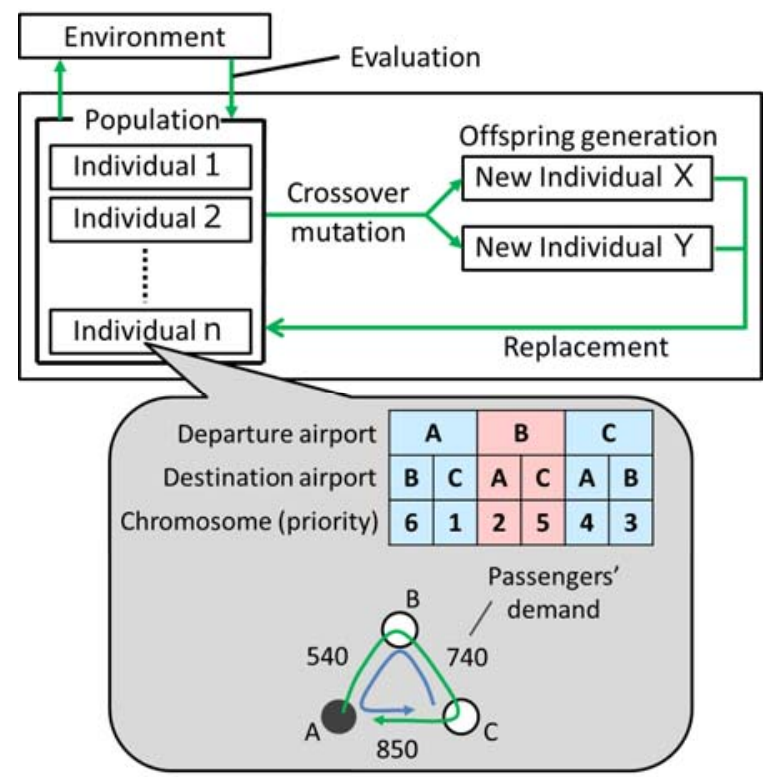

Among the several options of genetic operations in PriGA (Gen et al., 2008), the following two genetic operations are employed to avoid from duplicating the gene values in each individual.

\section{Position-based crossover}

The left side of Figure 5 shows the procedure of the position-based crossover operator. In this crossover, a subset of the genes of the first parent is copied to the offspring with a crossover rate of the corresponding locus of the offspring. For remaining empty locus of the offspring, a subset of the genes of the second parent is copied to the offspring.

Figure 5 (a) Position-based crossover (b) Swap mutation (see online version for colours)

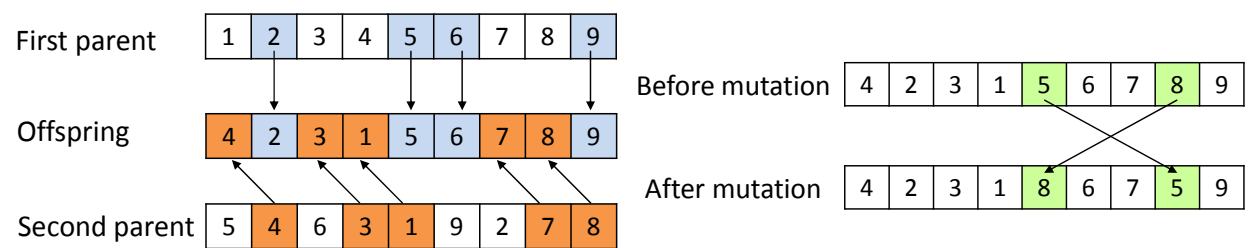

(a)

(b)

\section{Swap mutation}

The right side in Figure 5 shows the procedure of the swap mutation operator. In this mutation, for each individual, two loci are randomly chosen and two genes on the chosen loci are exchanged. 


\section{Proposed methods}

\subsection{MIN-/MAX-based fleet assignment method}

The proposed method is based on the conventional fleet assignment method (Gen et al., 2008) that assigns aircrafts to each flight route by considering passengers' demands of only one month. In the case of the fleet assignment by considering several months, aircrafts should be assigned to satisfy OD tables of all months. For example, a on-peak month and an off-peak month should be considered (two OD tables need to be satisfied in this case). However, the conventional fleet assignment method (Gen et al., 2008) cannot assign aircrafts for several months simultaneously.

Figure 6 Flight route example in off-peak and peak month (A-B-C-A) (see online version for colours)

\section{Off-peak month}
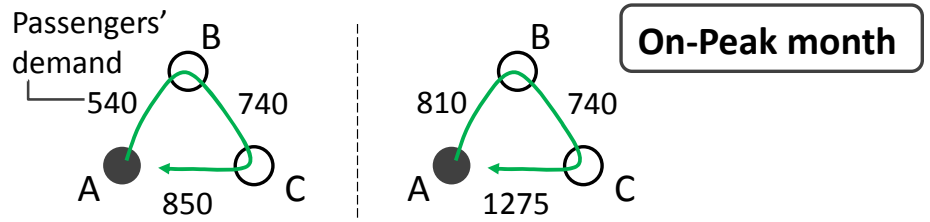

To overcome this problem, we propose the new fleet assignment method to assign aircrafts for both the regular and non-regular flights to a flight route by considering several months. Concretely, we propose two fleet assignment methods, the fleet assignment based on the maximum OD among all months and the fleet assignment based on the minimum OD among all months. Note that the way of route planning is the same as PriGA mechanism (Gen et al., 2008) described in Section 2. In the following sub-sub-sections, we explain the two proposed fleet assignment methods by using an example of a flight route shown in Figure 6. In this figure, the nodes and the arrow has the same meaning of Figure 2. What is the essential different between Figures 2 and 6 indicates the number of the months of the passengers' demands that have to be considered (i.e., one month in Figure 2 while two months in Figure 6). In Figure 6, the left side of this figure shows the passengers' demands between two airports for each airway in the off-peak month, while the right side of this figure shows the passengers' demands in the on-peak month.

\subsubsection{MIN-based fleet assignment method}

The MIN-based fleet assignment method assigns aircrafts to the routes in a flight network by considering the minimum passengers' demands. The procedure of the MIN-based fleet assignment method is summarised as follows:

1 For each airway, the passengers' demands between two months (e.g., the on- and off-peak months) are compared to determine the minimum one. For example, the left figure in Figure 7 shows a flight route including three airways and compares the passengers' demands between two months (e.g., 540 and 810 in the airway between $\mathrm{A}$ and $\mathrm{B}$ ) to select minimum one (i.e., 540). 
2 The maximum value among the minimum passengers' demands in all airways calculated in (1) is determined as MaxFlow. This process is the same as the conventional fleet assignment method (Gen et al., 2008) described in Section 2.2.2. For example, the maximum value (MaxFlow) among the minimum passengers' demands $(540,740$, and 850$)$ is 850 as shown in Figure 7.

3 The type and number of aircrafts for each flight route are assigned as the regular flights according to MaxFlow. This process is also the same as the conventional fleet assignment method (Gen et al., 2008). In this case, one large aircraft and two small aircrafts are assigned.

4 The non-regular flights for each flight route are assigned to satisfy all remaining passengers' demands which cannot be covered by the regular flights. As shown in Figure 8, when assigning one large aircraft and two small aircrafts (i.e., the total seats are 900) for the regular flights, the remaining passengers' demands of 375 persons still cannot be carried from the airport $A$ to $C$ in the on-peak month. The non-regular flights are assigned for such passengers' demands. Note that the non-regular flight is not assigned if the demand of all airways has been already satisfied with the regular flight.

Figure 7 Calculation example of MaxFlow for the MIN assignment method (see online version for colours)
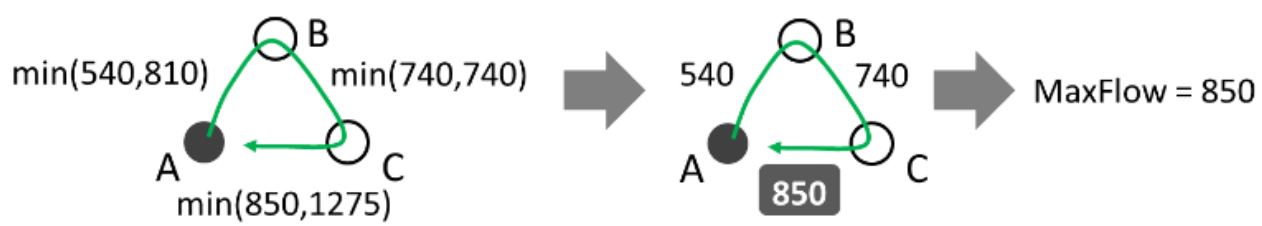

Figure 8 A flight route that cannot satisfy passengers' demands (see online version for colours)

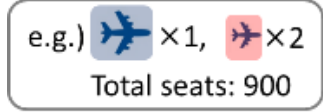

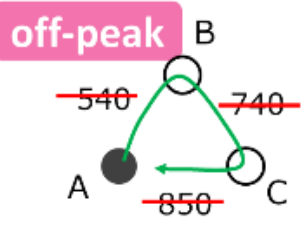

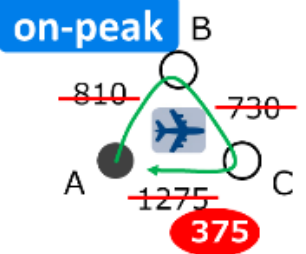

\subsubsection{MAX-based fleet assignment method}

The MAX-based fleet assignment method assigns aircrafts to the routes in a flight network by considering the maximum passengers' demand. The procedure of the MAX-based fleet assignment method is summarised as follows:

1 For each airway, the passengers' demands between two months (e.g., the on-and off-peak months) are compared to determine the maximum one. For example, the left figure in Figure 9 shows a flight route including three airways and compares the passengers' demands between two months (e.g., 540 and 810 in the airway between $\mathrm{A}$ and $\mathrm{B}$ ) to select maximum one (i.e., 810). 
2 The maximum value among the maximum passengers' demands in all airways calculated in (1) is determined as MaxFlow. For example, the maximum value among the maximum passengers' demands $(810,740$, and 1,275$)$ is 1,275 as shown the middle figure in Figure 9 and MaxFlow is set as 1,275 as shown the right figure in Figure 9.

3 The type and number of aircrafts for each flight route are assigned as the regular flights according to MaxFlow. Assuming that the large and small aircrafts, for example, the combinations of the fleet assignment that satisfy MaxFlow $(1,275)$ are five patterns as shown in the right-side table of Figure 10. After calculating the costs of all combinations, a combination of aircrafts that has the minimum cost is selected. In this case, two large aircrafts and two small aircrafts are assigned.

4 Since the regular flights are assigned according to the maximum demand of all airways, the capacity of the some assigned regular flight excessively exceeds the passengers' demand. In such a case, the excessively assigned regular flightsare changed into the non-regular flights. The route of the non-regular flights changed from the regular flight is inherited. As shown in Figure 11, when assigning two large aircrafts and two small aircrafts (i.e., the total seats are 1,300) for the regular flights, the seats are excessively assigned. Since the regular flights for the 450 seats at maximum can be removed in the off-peak month, these excessively assigned regular flights in for the on-peak month are changed into the non-regular flights. Note that the non-regular flight is not assigned if there is no excessively assigned regular flight in all flight routes.

Figure 9 Calculation example of MaxFlow for the MAX assignment method (see online version for colours)
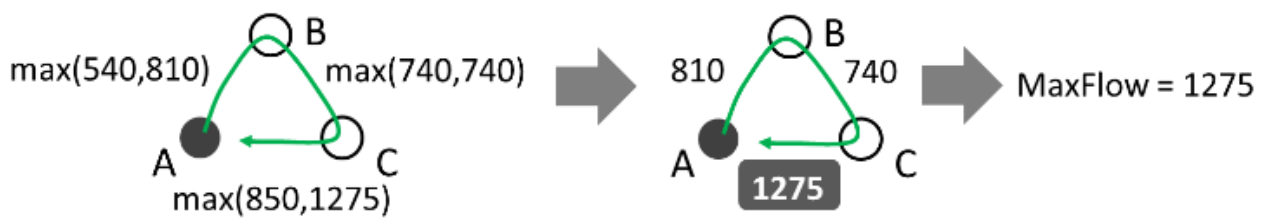

Figure 10 Example of the fleet assignment according to the MaxFlow (see online version for colours)

\begin{tabular}{c|c|c}
\hline $\begin{array}{c}\text { Type } \\
\text { of aircraft }\end{array}$ & Seats & Cost \\
\hline & 400 & 200 \\
\hline & 250 & 140 \\
\hline
\end{tabular}

\begin{tabular}{c|cccc}
\hline & Total & $\begin{array}{c}\text { Total } \\
\text { cost }\end{array}$ \\
\hline (1) & $\times 0$ & $\times 6$ & 1500 & 840 \\
(2) & $\times 1$ & $\times 4$ & 1400 & 760 \\
\hline (3) & $\times 2$ & $\times 2$ & 1300 & 640 \\
(4) & $\times 3$ & $\times 1$ & 1450 & 740 \\
(5) & $\times 4$ & $\times 0$ & 1600 & 800 \\
\hline
\end{tabular}


Figure 11 A excessively assigned seats against passengers' demands (see online version for colours)

e.g.) $\div \times 2, \% 2$ Total seats: 1300

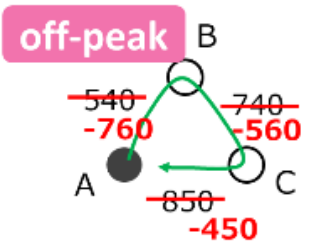

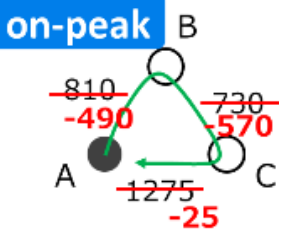

\subsubsection{Self-adaptive MIN-/MAX-based fleet assignment method}

For parameter and option setting in evolutionary algorithms (EAs), several approaches have been studied so far (Lobo et al., 2007). Generally, the deterministic approach employs a fixed parameters or options which are pre-determined before the solution search. To tune them, a number of algorithm runs with different parameters or options are needed, which is very time-consuming. The adaptive and self-adaptive approaches simultaneously optimise not only solutions but also parameters and options employed in EAs during the solutions in a single run of the algorithm.

Figure 12 Architecture of PriGA with CNM + MIN-/MAX-based fleet assignment (see online version for colours)

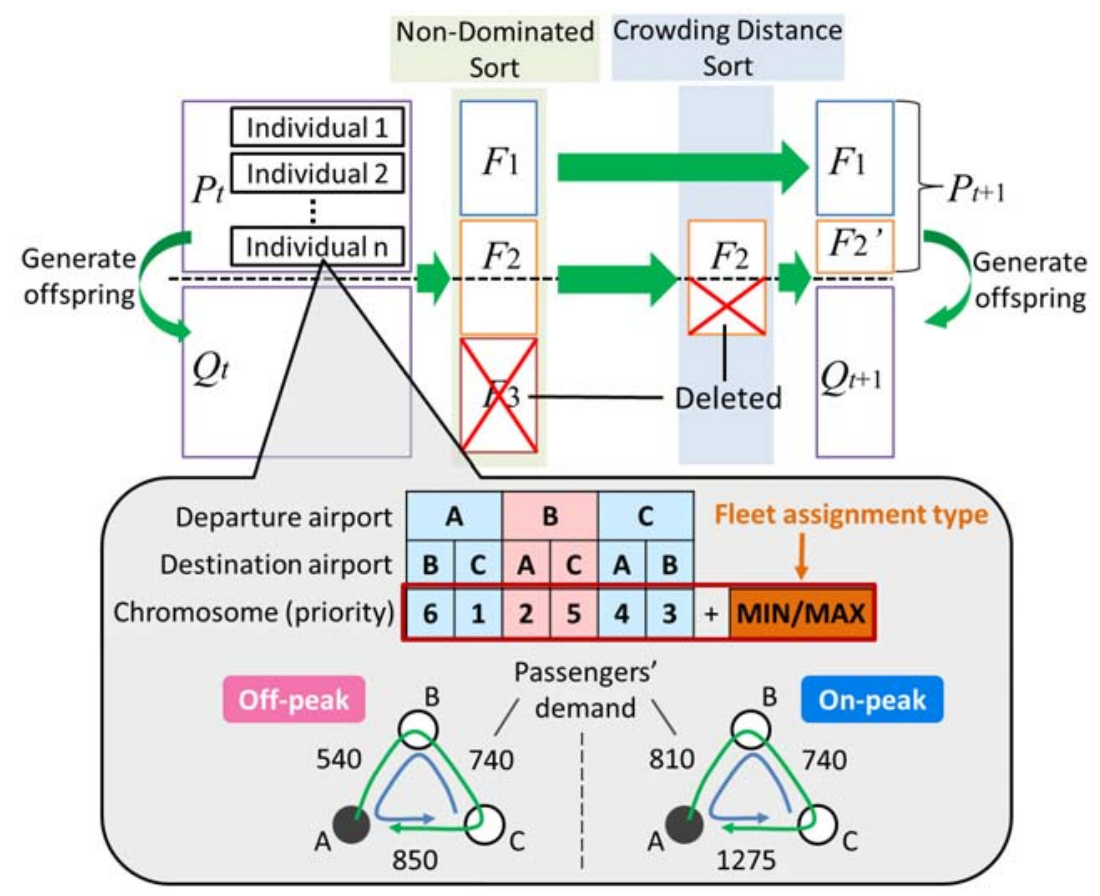

In this work, the proposed fleet assignment methods described above can determine either the MIN- or MAX-based fleet assignment 'deterministically' or 'self-adaptively'. Figure 12 shows the architecture of PriGA with CNM + MIN-/MAX-based fleet assignment, which indicates that the chromosome is composed of the original genes 
determining the priority with the specific gene determining the type of the fleet assignment (i.e., the MIN or MAX assignment). Note that the gene determining MIN-/MAX-based fleet assignment is fixed in the deterministic case, while it can be varied in the evolution process in the self-adaptive case. Concretely, in the deterministic case, the MIN-/MAX-based assignment method needs to be pre-determined before the solution search, and the same assignment method is applied for all solutions in the population during the solution search. On the other hand, in the self-adaptive case, both original and the specific genes are varied to automatically find the appropriate assignment type for each solution during the solutions search.

The proposed method is based on elitist non-dominated sorting genetic algorithm II (NSGA-II) (Deb et al., 2002) as one of major multi-objective evolutionary algorithms (MOEAs) (Deb, 2001; Coello et al., 2007) and its algorithm is summarised as follows:

1 The initial population is randomly generatedas $P_{t}$ while avoiding the duplication of gene values in each individual.

2 All individuals in the population $P_{t}$ are decoded to generate flight networks and their fitness of both regular and non-regular flights are calculated (see in Section 5.2.2).

3 The offspring $Q_{t}$ are generated by applying the crossover operator, and the mutation operator is conducted with a predetermined mutation rate. Concretely, two individuals are randomly chosen from the parent population $P_{t}$, and the individual with a higher non-dominance rank is selected as the parent. If two individuals have the same non-dominance rank, the individual with a larger crowding distance is selected as the parent. This offspring generation is repeated until the size of $Q_{t}$ becomes that of $P_{t}$.

4 All individuals are classified into the several non-dominance ranks $F_{1}, F_{2} \ldots$ by the non-dominated sort.

5 The half size of all individuals $\left(P_{t}+Q_{t}\right)$ are selected as the parent population $P_{t+1}$. Note that when the total individual size exceeds the half size of $P_{t}+Q_{t}$ by adding individuals in a certain $F\left(F_{2}\right.$ in Figure 12), the individuals with a large crowding distance in a certain $F$ are selected as the parent population $P_{t+1}$. This selection is repeated until the total individual size becomes the half size of $P_{t}+Q_{t}$.

6 The steps (2)-(5) are repeated until the predetermined termination condition is satisfied.

\subsection{Weight-based fleet assignment method}

\subsubsection{Deterministic weight-based fleet assignment method}

The MIN-/MAX-based assignment methods described in Section 3.1 assign aircrafts by considering the demand of either on- or off-peak month. However, a flight network generated by the MIN-based assignment method achieves a high profit in the off-peak month but its profit in the on-peak month becomes low. In contrast, a flight network generated by the MAX-based assignment method achieves a high profit in the on-peak month but its profit in the off-peak month becomes low. This indicates that the MIN-/MAX-based assignment method only optimise the specialised flight network for 
the on- or off-peak month, which makes it difficult to obtain flight networks which achieve well-balanced profits between the on- and off-peak months.

To tackle this problem, we propose the weight-based fleet assignment method. Since this method assigns the regular flights based on MaxFlow discounted by the weight factor, this method slightly decreases the number of regular flights for the given passengers' demands, which can assign different non-regular flights for each month. If the same non-regular flights exist in all months, these flights are regarded as regular flights because they are common flights in all months.

\subsubsection{Self-adaptive weight-based fleet assignment method}

Similar to the self-adaptive MIN-/MAX-based fleet assignment method, the weight value employed in the weight-based fleet assignment method can also be determined 'deterministically' or 'self-adaptively'. Figure 13 shows the architecture of PriGA with $\mathrm{CNM}+$ weight-based fleet assignment, which indicates that the chromosome is composed of the original genes determining the priority with the specific gene determining the weight factor for MaxFlow. Note that the gene determining the weight factor is fixed in the deterministic case, while it can be varied in the evolution process in the self-adaptive case. Concretely, in the deterministic case, the weight factor needs to be pre-determined before the search and the same weight value is used for all solutions in the population during the solution search. On the other hand, in the self-adaptive case, both original and specific genes are varied to automatically find the appropriate weight value for each solution during the solutions search. The proposed method follows the same procedure described in Section 3.1.3.

Figure 13 Architecture of PriGA with CNM + weight-based fleet assignment (see online version for colours)

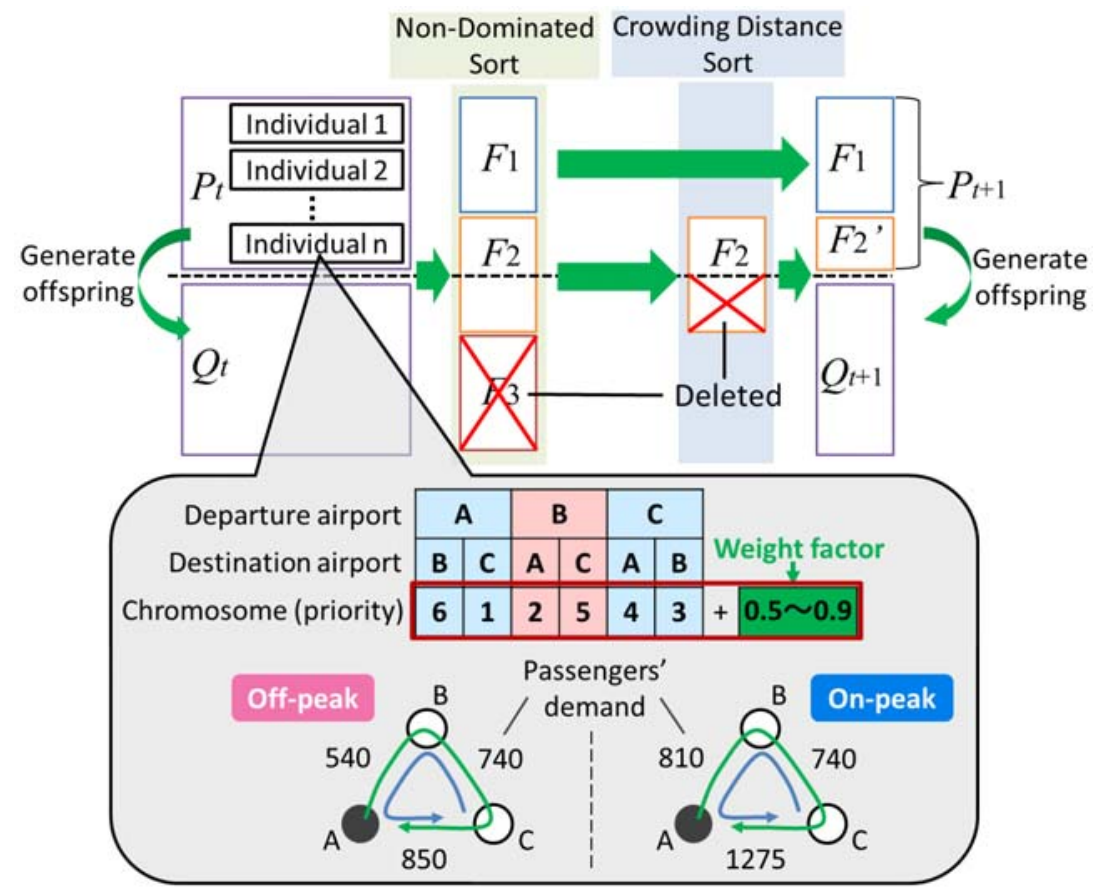




\subsection{Double self-adaptive fleet assignment method: self-adaptive min-/max-based and weight-based fleet assignment methods}

By integrating the self-adaptive MIN-/MAX-fleet assignment method with the self-adaptive weight-based fleet assignment, all values (i.e., the original genes for priority of the route in PriGA, the gene for determining the type of fleet assignment, and the gene for the weight value) can be evolved during the solutions search. Figure 14 shows the architecture of PriGA with CNM + MIN-/MAX-based fleet assignment + weight-based fleet assignment, which indicates that the chromosome is composed of the original genes determining the priority with the specific genes determining the type of the fleet assignment (i.e., the MIN or MAX assignment) and the weight factor for MaxFlow. The appropriate these values for each solution are automatically found during the solution search. The proposed method follows the same procedure described in Section 3.1.3.

Figure 14 Architecture of PriGA with CNM + MIN-/MAX-based fleet assignment + weight-based fleet assignment (see online version for colours)

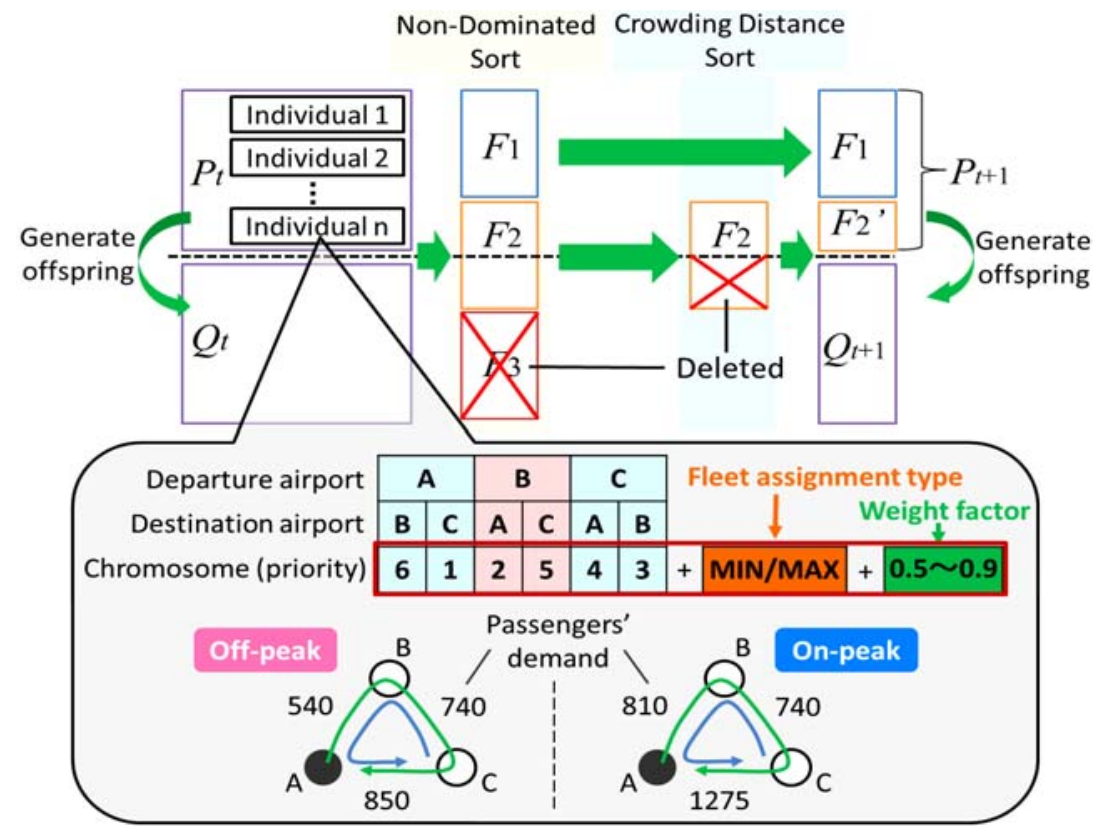

\section{Problem description: domestic airport network based on CNM}

To investigate the effectiveness of the proposed methods, we apply them to the domestic airport network problem. Concretely, this paper focuses on a five Japanese domestic airport network based on the CNM as shown in Figure 15. In this figure, the nodes and the arrows indicate airports and airways, respectively. In this network, Nagoya airport is connected only with Fukuoka as the same as the real-world flight network. Although this network consists of five airport, it is difficult to find the optimal solution (i.e., flight network) because its search space is still large (i.e., the search space is 14 ! combinations because of 14 airways). 
Figure 15 Flight network of the test problem

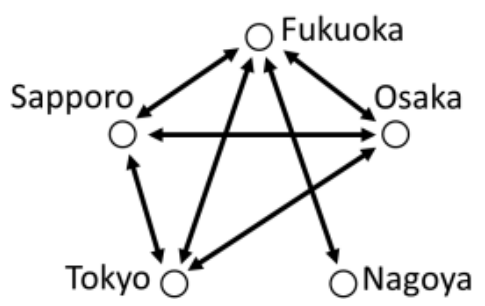

Table 3 Daily OD tables in off-peak and on-peak month

\begin{tabular}{|c|c|c|c|c|c|c|c|c|c|c|c|}
\hline & \multicolumn{5}{|c|}{ Off-peak } & \multicolumn{6}{|c|}{ On-peak } \\
\hline & $\frac{\frac{D}{2}}{\frac{\pi}{3}}$ & $\begin{array}{l}\frac{\pi}{3} \\
\frac{3}{2} \\
0\end{array}$ & 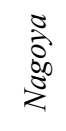 & $\frac{8}{0}$ & 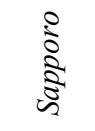 & & $\frac{\frac{\mathfrak{D}}{3}}{\frac{\pi}{3}}$ & $\begin{array}{l}\frac{\pi}{2} \\
0 \\
0\end{array}$ & 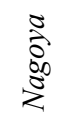 & $\frac{0}{2}$ & $\begin{array}{c}0 \\
\vdots \\
\vdots \\
\vdots \\
5\end{array}$ \\
\hline Fukuoka & 0 & 764 & 102 & 4,235 & 96 & Fukuoka & 0 & 774 & 161 & 4,790 & 360 \\
\hline Osaka & 764 & 0 & 0 & 3,603 & 177 & Osaka & 774 & 0 & 0 & 3,788 & 585 \\
\hline Nagoya & 102 & 0 & 0 & 0 & 0 & Nagoya & 161 & 0 & 0 & 0 & 0 \\
\hline Tokyo & 4,235 & 3,603 & 0 & 0 & 3,491 & Tokyo & 4,790 & 3,788 & 0 & 0 & 6,211 \\
\hline Sapporo & 96 & 177 & 0 & 3,491 & 0 & Sapporo & 360 & 585 & 0 & 6,211 & 0 \\
\hline
\end{tabular}

To consider the on- and off-peak seasons, this paper optimises the flight network according to two months, i.e., April (the off-peak season) and August (the on-peak season). Table 3 shows daily OD tables for the off-peak month (left) and on-peak month (right), respectively. In this table, the column and row indicate the departure airports and the destination ones, respectively, and the OD data is based on data of All Nippon Airways (ANA) website in Japan (ANA Japan, http://www.ana.co.jp/). In this table, zero means that no flight exists between these two airports.

Table 4 Aircraft's data

\begin{tabular}{lccc}
\hline Type of aircraft & Large & Medium & Small \\
\hline Seats & 495 & 267 & 143 \\
Total number of flights (off-peak) & 79 & 29 & 35 \\
Total number of flights (on-peak) & 82 & 28 & 34 \\
\hline
\end{tabular}

Note: The number of seat and constraints of flight's count.

In this airport network problem, three types of aircraft are employed as shown in Table 4. Concretely, aircraft types are simplified into three types, small, medium and large. The boarding rates for each month determined by data from ANA's website (ANA Japan, http://www.ana.co.jp/) is employed to estimate the real number of the seats of each aircraft. For example, if the maximum number of the seats of an aircraft $X$ is 100 and the boarding rate is 0.5 , the estimated real number of the seats the aircraft $X$ is 50 persons. The boarding rate is different according to month. The total number of flights shown in Table 4 is also based on the timetable of ANA as a constraint. Each type of aircraft has to be assigned less than the total number of flights. If the total number of some flight routes 
exceeds this constraint, the flight network including such routes is treated as the infeasible solution.

\section{Experiment}

\subsection{Overview of experiments}

Table 5 shows the overview of our experiments. In Table 5, the column indicates the experiment cases, while the row indicates whether the fleet assignment type and/or the weight factor are determined 'deterministically' and 'self-adaptively'. Regarding the fleet assignment type, the MIN-/MAX-based fleet assignment type is fixed (or pre-determined beforehand) in the deterministic fleet assignment method (in the experiment 1), while it is determined automatically in the self-adaptive fleet assignment method (in the experiments 2 and 3). Regarding the weight factor, on the other hand, the weight value is fixed (or pre-determined beforehand) as the deterministic weight factor (in the experiments 1 and 2), while it is determined automatically as the self-adaptive weight factor (in the experiments 3 ). Note that the weight value is represented as a discrete value from 0.5 to 1.0 .

Table 5 Overview of experiments

\begin{tabular}{lcl}
\hline & Fleet assignment & Weight \\
\hline Experiment 1 & Deterministic & Deterministic \\
Experiment 2 & Self-adaptive & Deterministic \\
Experiment 3 & Self-adaptive & Self-adaptive \\
\hline
\end{tabular}

\subsection{Experiment 1}

\subsubsection{Comparison}

The experiment 1 compares the following four methods shown in Table 6 . In this table, the column indicates the compared methods, while the row indicates the type of the method (i.e., the proposed or conventional method), a method label for distinguishing the methods with different parameter setting, the type of a fleet assignment and a weight factor. In this experiment, both the fleet assignment type and the weight factor are pre-determined beforehand (i.e., they do not vary in the evolution process). Since the conventional method (PriGA) can only optimise the flight network from the viewpoint of the single-objective optimisation, it optimises the flight network of either the on- or off-peak month. The methods $1 \mathrm{~A}$ and $1 \mathrm{~B}$ are respectively the MIN-and MAX-based fleet assignment method (with 1.0 weight factor).

Table 6 Comparison

\begin{tabular}{lccc}
\hline Proposed/conventional & Method & Fleet assignment method & Weight factor \\
\hline Conventional (Pri-GA) & ---- & Optimise off-peak month only & ---- \\
& ---- & Optimise on-peak month only & ---- \\
Proposed & 1A & Deterministic (MIN) & Deterministic (1.0) \\
& 1B & Deterministic (MAX) & Deterministic (1.0) \\
\hline
\end{tabular}




\subsubsection{Evaluation criteria and parameters}

As the evaluation criteria, our experiment employs the profit of a daily flight network in the on- and off-peak months as shown in the following equation.

$$
\begin{aligned}
& \text { profit }=(\text { sale })-(\text { cost }) \\
& \text { sale }=(\text { fare }) \times(\text { numberof passenger }) \\
& \text { cost }=(\text { transportation cost of airline })
\end{aligned}
$$

From the above equation, we define the profit (i.e., the fitness) of a flight network generated by the proposed methods as the sale minus cost of airline. The sale of airline is calculated by multiplying a fare of each airway by the number of passengers, and the fare is employed from the ANA's website in Japan (ANA Japan, http://www.ana.co.jp/). The transportation costs of each airway and each type of aircraft are calculated by the cost of fuel in the relevant study (Inoue, 2012). Note that we do not consider a discounted service of the fare as the first step of this research.

Table $7 \quad$ Experimental parameters

\begin{tabular}{lccc}
\hline Population size & Generation & \multicolumn{2}{c}{ Parent selection } \\
\hline 200 & 3,000 & Tournament selection \\
\hline Crossover & Mutation & Crossover rate & Mutation rate \\
\hline Position-based crossover & Swap mutation & 0.5 & 0.5 \\
\hline
\end{tabular}

Regarding the parameter setting, Table 7 shows the experimental parameters which are common in the conventional and proposed methods. Concretely, a population size as the number of individuals is set as 200, the number of generations is set as 3000 , the position-based crossover as the crossover operation and the swap mutation as the mutation operation are employed, their rate are respectively set as 0.5 and 0.5 , and the tournament selection is employed to select parents.

\subsubsection{Results}

Figure 16 shows the solutions optimised by the conventional and proposed methods in Table 6, where the horizontal and vertical axes indicate the profit of the on- and off-peak months respectively. Note that the plots indicate the evolved solutions (i.e., the flight network) and this figure only shows the solutions of the Pareto front 1 . In detail, the dotted lines in this figure indicate the profit of the flight network of on-/off-peak month separately optimised by the conventional method, PriGA. The square marks with the blue background colour and the diamond marks with the pink background colour indicate the profit of the flight network optimised by the MIN-and MAX-based fleet assignment methods, respectively. Note that the proposed methods can optimise the flight network from the viewpoint of both on- and off-peak months unlike the conventional method. 
Figure 16 Solutions evolved by methods 1A and 1B (see online version for colours)

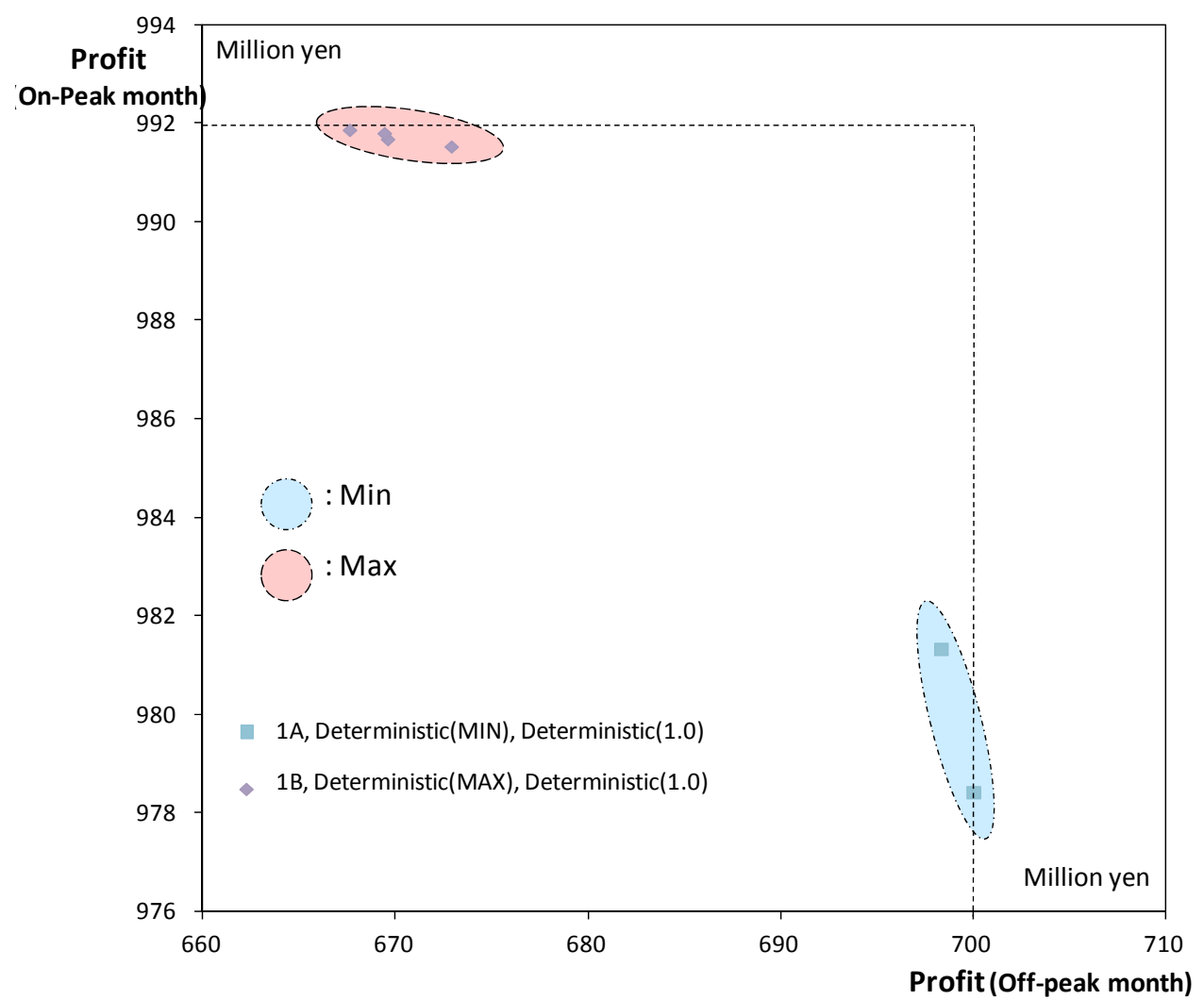

Focusing on the distribution of the solutions optimised by the proposed methods, the solutions of the MIN-based fleet assignment method are distributed in the lower-right side of this figure (i.e., these solutions have the high profit in the off-peak month), while those of the MAX-based fleet assignment method are distributed in the upper-left side of this figure (i.e., these solutions have the high profit in the on-peak month). This is because the MIN-/MAX-based fleet assignment methods start to assign the regular flights considering the minimum/maximum number of the passengers, which is respectively suit for the off-/on-peak month.

\subsubsection{Discussion}

\section{- Feasibility of solutions and their limitation}

The solutions evolved for either on- or off-peak month by the conventional method, PriGA, cannot be applied into the opposite month (i.e., the flight networks optimised for the on-peak month cannot be applied into the off-peak month and vice versa). In comparison with these solutions, those evolved by the proposed method (i.e., methods $1 \mathrm{~A}$ and 1B) can be applied into both the on- and off-peak month. This is a clear advantage of the proposed method.

As described in Section 5.2.3, however, a flight network evolved by the method 1A (i.e., the MIN-based fleet assignment method) is specialised to the off-peak month, while 
one evolved by the method 1B (i.e., the MAX fleet assignment method) is specialised to the on-peak month as shown in Figure 16. This suggests that the proposed method $1 \mathrm{~A}$ and $1 \mathrm{~B}$ has a limitation of finding the solutions having the well-balanced profit between two months.

\subsection{Experiment 2}

\subsubsection{Comparison, evaluation criteria, and parameters}

The experiment 2 compares the following eight methods shown in Table 8. In this table, the column and row have the same meaning of Table 6. Concretely, the conventional methods (PriGA) for on-/off-peak month are the same ones employed in the experiment 1 . For the methods $2 \mathrm{~A}-2 \mathrm{~F}$, the fleet assignment type (MIN or MAX) is self-adaptively determined through the evolution process while the weight factors are fixed as $\{1.0,0.9,0.8,0.7,0.6$, and 0.5$\}$ to investigate the effects of weight factor.

Table 8 Comparison

\begin{tabular}{lccc}
\hline Proposed/conventional & Method & Fleet assignment method & Weight factor \\
\hline Conventional (Pri-GA) & ---- & Optimise off-peak month only & --- \\
& ---- & Optimise on-peak month only & --- \\
Proposed & 2A & Self-adaptive (MIN/MAX) & Deterministic (1.0) \\
& 2B & Self-adaptive (MIN/MAX) & Deterministic (0.9) \\
& 2C & Self-adaptive (MIN/MAX) & Deterministic (0.8) \\
& 2D & Self-adaptive (MIN/MAX) & Deterministic (0.7) \\
& 2E & Self-adaptive (MIN/MAX) & Deterministic (0.6) \\
& 2F & Self-adaptive (MIN/MAX) & Deterministic (0.5) \\
\hline
\end{tabular}

Regarding the evaluation criteria and common parameters of the conventional and proposed methods, we employ the same evaluation criteria and parameters described in the experiment 1 . For the methods $2 \mathrm{~A}-2 \mathrm{~F}$, however, the mutation rate for the fleet assignment method is additionally set as shown in Table 9.

Table 9 Experimental parameters

\begin{tabular}{l}
\hline Mutation rate (fleet assignment method) \\
\hline 0.3 \\
\hline
\end{tabular}

Note: 'Self-adaptive case' only.

\subsubsection{Results}

Figure 17 shows the solutions optimised by the conventional and proposed methods shown in Table 8. The horizontal and vertical axes and the dotted lines have the same meaning of Figure 16. As the same as the result in Figure 16, Figure 17 only shows the solutions (i.e., the flight network) of the Pareto front 1. In detail, the (blue) square marks, (green) triangle marks, (purple) cross marks, (light blue) asterisk marks, (orange) circle marks, and (light purple) plus marks respectively indicate the profit of the flight network optimised by the proposed methods $2 \mathrm{~A}, 2 \mathrm{~B}, 2 \mathrm{C}, 2 \mathrm{D}, 2 \mathrm{E}$, and $2 \mathrm{~F}$, i.e., the self-adaptive MIN-/MAX-based fleet assignment method using the weight factors fixed as $\{1.0,0.9$, 
$0.8,0.7,0.6$, and 0.5$\}$. Since the proposed methods from $2 \mathrm{~A}$ to $2 \mathrm{~F}$ self-adaptively determines the fleet assignment type (MIN or MAX), the background colour of the solutions becomes either blue or pink according to the evolved gene of the fleet assignment type. For example, the gene of the fleet assignment type in the solutions represented by the (blue) square marks in the lower-right side is evolved as the MIN-based fleet assignment, providing the blue background colour. The gene in the solutions represented by the same (blue) square marks in the upper-left side, on the other hand, is evolved as the MAX-based fleet assignment, providing the pink background colour.

Figure 17 Solutions evolved by methods from $2 \mathrm{~A}$ to $2 \mathrm{~F}$ (see online version for colours)

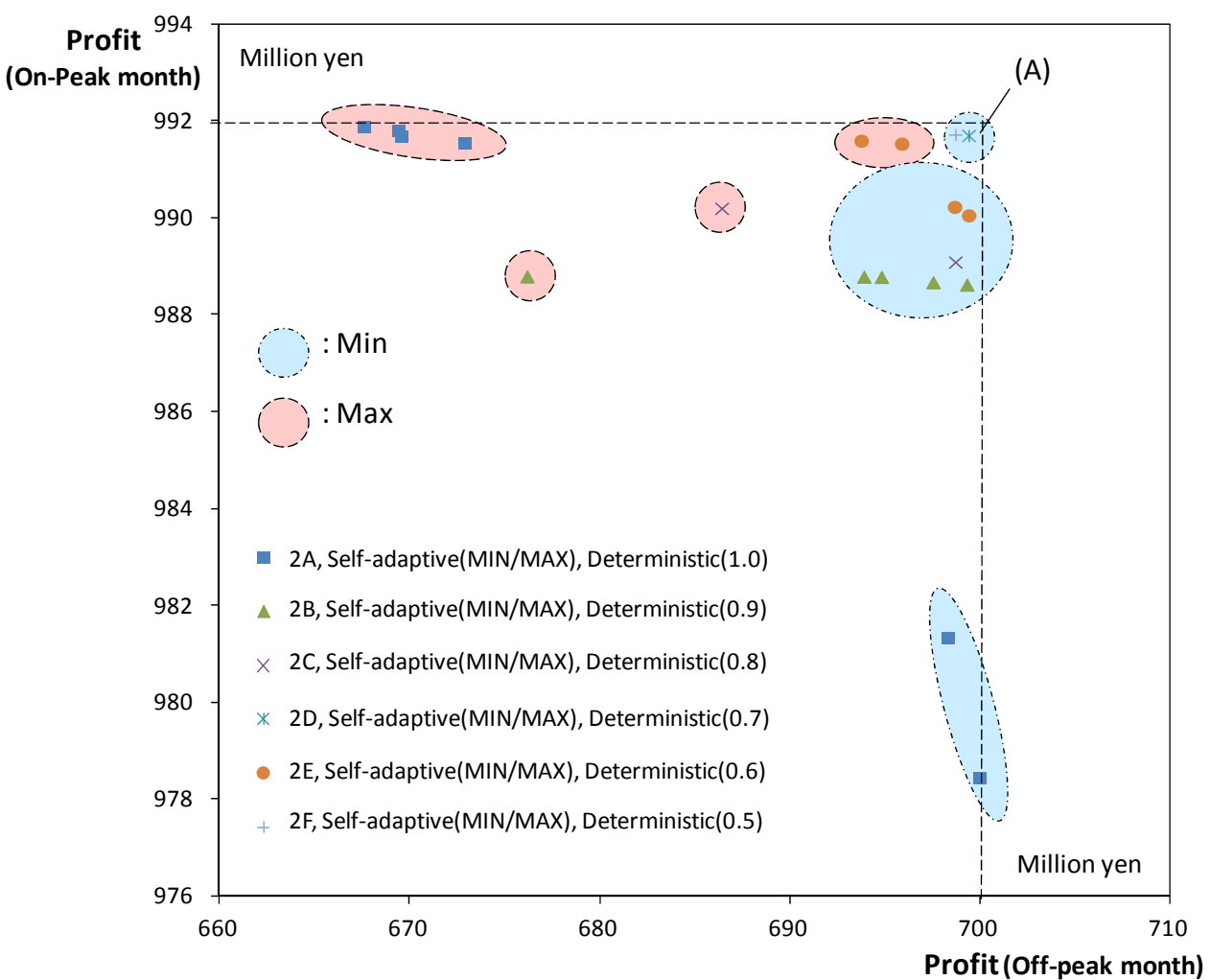

From Figure 17, the following implications have been found:

1 Comparing with the result of the experiment 1 , the solutions evolved by the methods $1 \mathrm{~A}$ and $1 \mathrm{~B}$ are the same as those evolved by the method $2 \mathrm{~A}$. This indicates that the self-adaptive MIN-/MAX-based fleet assignment method can provide both solutions of the deterministic MIN-/MAX-based fleet assignment.

2 Among the solutions evolved by the method from $2 \mathrm{~A}$ to $2 \mathrm{~F}$ in Figure 17, the method $2 \mathrm{D}$ (represented (A) in this figure) can provide the well-balanced solutions between the on- and off-peak months. 
3 Focusing on the distribution of the solutions optimised by the proposed methods from $2 \mathrm{~B}$ to $2 \mathrm{~F}$, the solutions are distributed not only in the both sides of the Pareto front (i.e., such solutions are specialised for one month), but also in the centre of the Pareto front (i.e., such solutions have a good balance between the on- and off-peak months).

\subsubsection{Discussion}

\section{- Effect of weight factor}

Since the number and/or size of the regular flights becomes small by assuming the slightly smaller passengers' demand when the weight factor is less than 1.0, the several non-regular ones can be assigned to each month to satisfy the passengers' demand that cannot be satisfied by the regular flights. Such an insertion of the several non-regular flights increases the diversity of the flight network in comparison with that of PriGA as the conventional method. This diversity of solutions is indispensable to explore the solution space, which contributes to finding the solutions in the upper-right corner shown in Figure 17.

For example, the well-balanced solutions between the on- and off-peak months are acquired in the case of the weight factors set from 0.5 to 0.7 in comparison with the solutions in the case of the weight factors set as 0.8 or higher. This is simply because the ratio of the non-regular flights in the former case is larger than that in the latter case, which increases the diversity of the flight network. This implication suggests an importance of the weight factor to explore the well-balanced solutions.

\section{- $\quad$ Flight network affected by weight factor}

Figure 18(a) shows the flight network optimised by the method 2D (with the 0.7 weight value) that has the highest sum profit of the on- and off-peak months (i.e., the solution (A) in Figure 17), while Figure 18(b) shows the flight network optimised by the method $2 \mathrm{~A}$ (with the 1.0 weight value). In Figures 18(a) and 18(b), the left figure indicates the regular flight network, while the upper-right and lower-right figures indicate the non-regular one of the off- and on-peak months, respectively. The nodes and the arrows indicate the airports and the flight route, respectively.

Specifically, focusing on the non-regular flight route between Tokyo and Fukuoka, the four large airplanes are assigned in the off-peak month while the six medium airplanes are assigned in the on-peak month in Figure 18(a). In contrast, the three small airplanes are only assigned in the off-peak month while 'none' of airplanes are assigned in the on-peak month in Figure 18(b). This indicates that the method 2D (with the 0.7 weight value which is less than 1.0) can assign the different number and size of the non-regular flights according to the on- or off-peak month, while the method 2A (with the 1.0 weight value) can only assign the non-regular flights either on- or off-peak month. This is because the several non-regular flights can be assigned to each month when the weight factor is less than 1.0 as described in the previous discussion. 
Figure 18 Flight network optimised by the methods 2D and 2A, (a) flight network optimised by the method 2D (b) flight network optimised by the method 2A (see online version for colours)
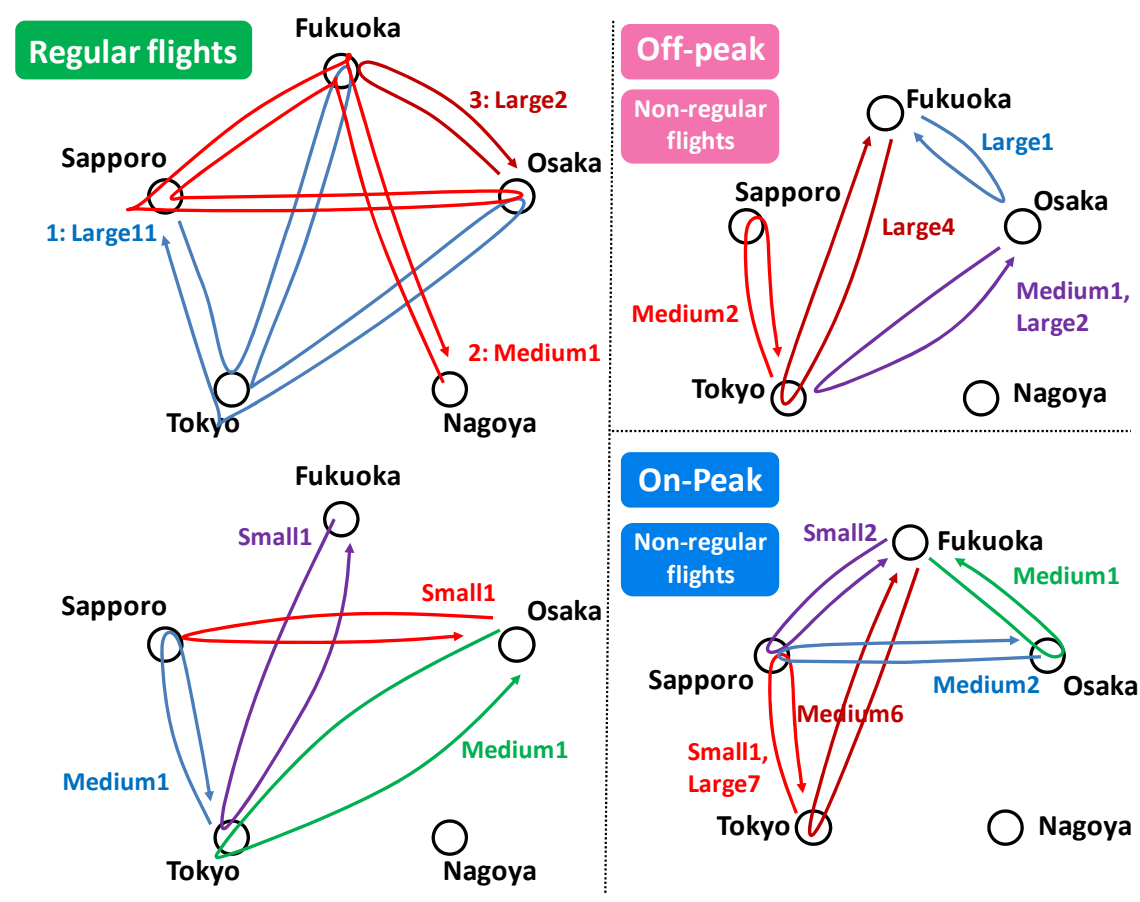

\section{On-Peak}

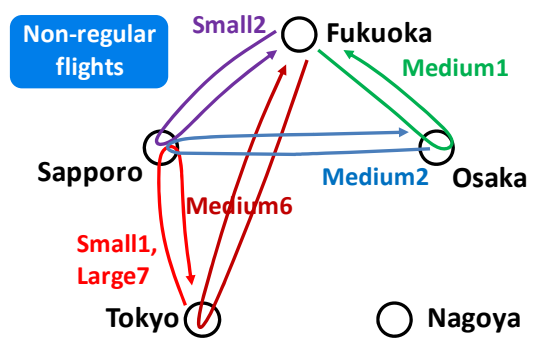

(a)
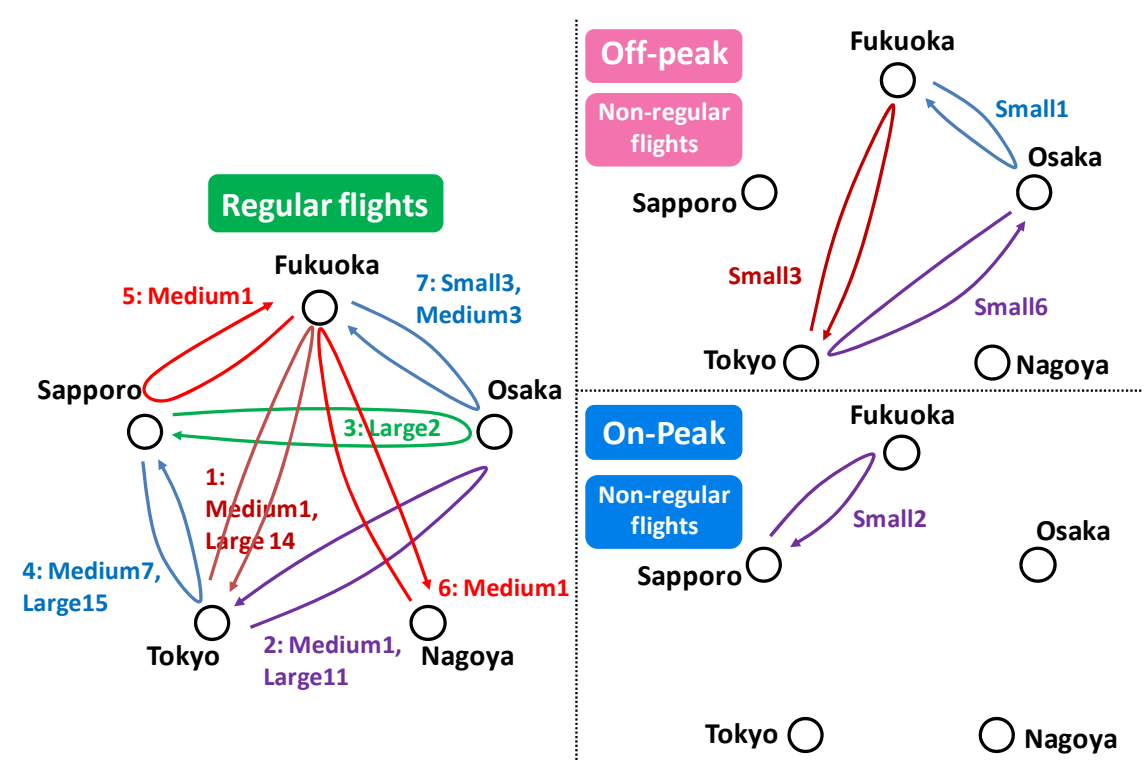

(b)

Note: The solution (A) in Figure 17. 


\subsection{Experiment 3}

\subsubsection{Comparison, evaluation criteria, and parameters}

The experiment 3 compares the following nine methods shown in Table 10. In this table, the column and row have the same meaning of Table 6 . Concretely, the conventional methods (PriGA) for on-/off-peak month and the methods from 2A to $2 \mathrm{~F}$ are the same ones employed in the experiments 1 and 2. For the method 3, both the fleet assignment type (MIN or MAX) and weight factors (from 0.5 to 1.0 ) are self-adaptively determined through the evolution process.

Table 10 Comparison

\begin{tabular}{lccl}
\hline Proposed/conventional & Method & Fleet assignment method & Weight factor \\
\hline Conventional (Pri-GA) & ---- & Optimise off-peak month only & ---- \\
& ---- & Optimise on-peak month only & ---- \\
Proposed & 2A & Self-adaptive (MIN/MAX) & Deterministic (1.0) \\
& 2B & Self-adaptive (MIN/MAX) & Deterministic (0.9) \\
2C & Self-adaptive (MIN/MAX) & Deterministic (0.8) \\
2D & Self-adaptive (MIN/MAX) & Deterministic (0.7) \\
& 2E & Self-adaptive (MIN/MAX) & Deterministic (0.6) \\
& 2F & Self-adaptive (MIN/MAX) & Deterministic (0.5) \\
& 3 & Self-adaptive (MIN/MAX) & Self-adaptive (interval: 0.01) \\
\hline
\end{tabular}

Regarding the evaluation criteria and common parameters of the conventional and proposed methods, we employ the same evaluation criteria and parameters described in the experiments 1 and 2. For the method 3, two mutation rates for the fleet assignment method and the weight factor are set as in Table 11.

Table 11 Experimental parameters

\begin{tabular}{lc}
\hline Mutation rate (fleet assignment method) & Mutation rate (weight factor) \\
\hline 0.3 & 0.3 \\
\hline
\end{tabular}

Note: 'Self-adaptive case' only.

\subsubsection{Results}

Figure 19 shows the solutions optimised by the conventional and proposed methods shown in Table 10. In particular, Figure 19(b) shows the enlarge view of the upper-right of Figure 19(a). The horizontal and vertical axes, the dotted lines, and the six marks of the methods from $2 \mathrm{~A}$ to $2 \mathrm{~F}$ (i.e., the (blue) square marks, (green) triangle marks, (purple) cross marks, (light blue) asterisk marks, (orange) circle marks, and (light purple) plus marks) have the same meaning of Figure 17. As the same as the result in Figures 16 and 17, Figure 19 only shows the solutions (i.e., the flight network) of the Pareto front 1 . In detail, the (blue) bar marks indicate the profit of the flight network optimised by the proposed method 3, i.e., the integration of the self-adaptive MIN-/MAX-based fleet assignment method and the self-adaptive weight-based fleet assignment method. In particular, the method 3 evolves three solutions of the Pareto front 1, i.e., two solutions 
evolved in the upper-right corner and one solution evolved in the lower-right side in Figure 19(a).

From Figures 19(a) and 19(b), the following implications have been found:

1 the solution $\left(\mathrm{C}_{2}\right)$ evolved by the method 3 in the upper-right corner dominates all other solutions evolved by the other methods as shown in Figures 19 [note that the solutions (C2) and (2D) show a potential of earning approximately 992 and 700 million yen in the on-peak and off-peak months, but the solution $\left(\mathrm{C}_{2}\right)$ dominates the solution (2D) evolved by the method 2D as the best solution among the methods from $2 \mathrm{~A}$ to $2 \mathrm{~F}$ from Figure 19(b)]

2 focusing on the on-peak month (i.e., the vertical axis) in Figure 19(b), the method 3 can evolve the solution $\left(\mathrm{C}_{1}\right)$ that has higher profit (approximately 0.2 million yen) than the solution (B) evolved by PriGA as the conventional method even though the method 3 has to consider two months while the conventional method only considers a single month in the flight network optimisation.

Figure 19 Solutions evolved by methods from 2A to 2F and method 3, (a) total view (b) enlarged view (see online version for colours)

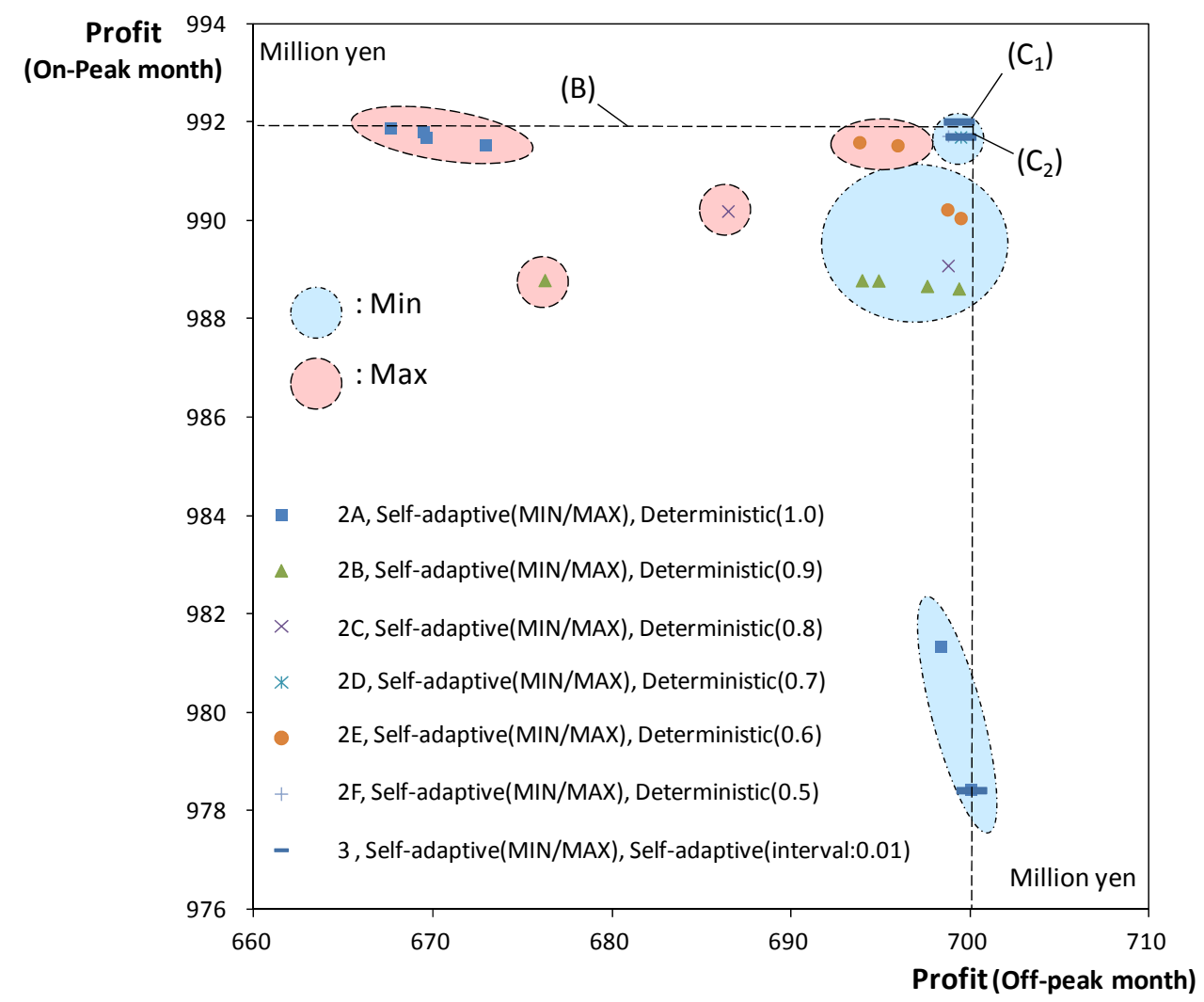

(a) 
Figure 19 Solutions evolved by methods from $2 \mathrm{~A}$ to $2 \mathrm{~F}$ and method 3, (a) total view (b) enlarged view (continued) (see online version for colours)

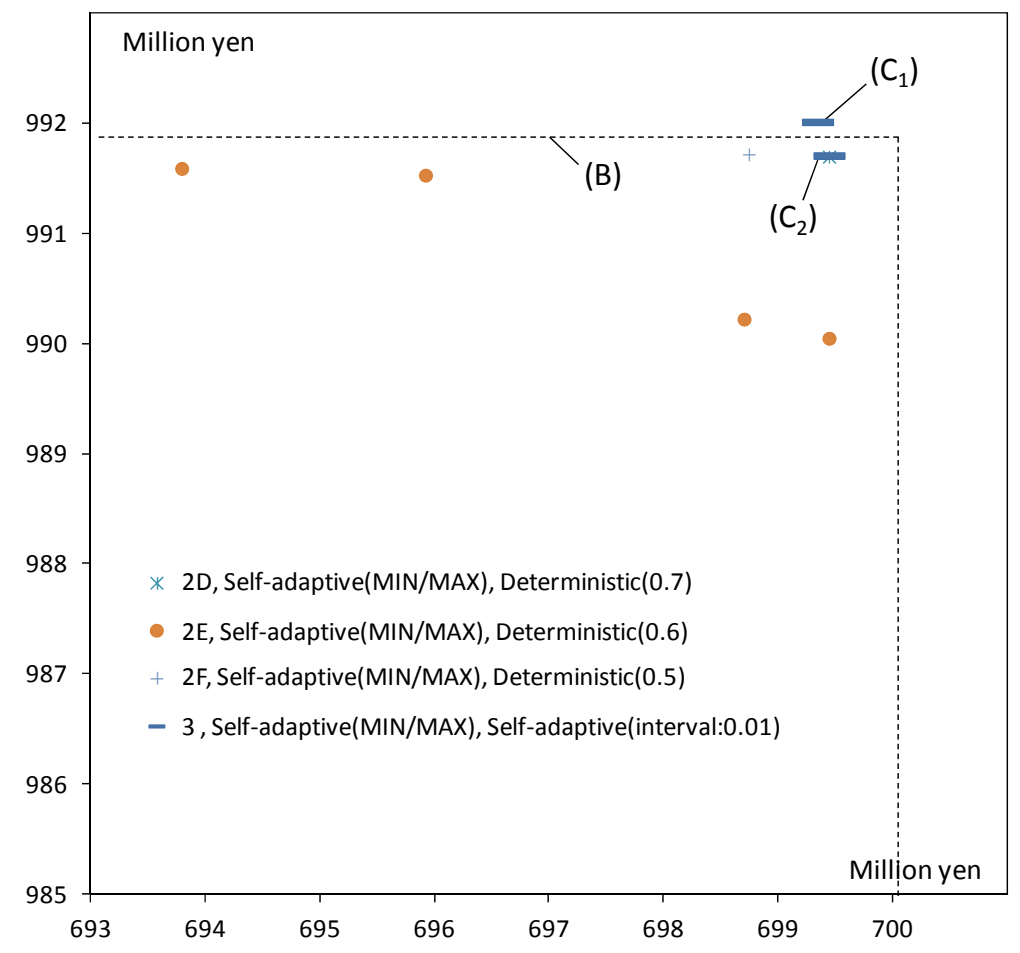

(b)

\subsubsection{Discussion}

- Fine search by double self-adaptive fleet assignment method

The method 3 finds three non-dominated solutions, and their weight factors determined through the self-adaptive evolutionary process are $\{0.85,0.58$, and 1.00$\}$, respectively. Considering the fact that the solution $\left(\mathrm{C}_{2}\right)$ with the 0.58 weight factor evolved by the method 3 in the upper-right corner dominates all other solutions evolved by the other methods as shown in Figure 19, the double self-adaptive fleet assignment method (i.e., the integration of the self-adaptive MIN-/MAX-based fleet assignment method and the self-adaptive weight-based fleet assignment method) has a great potential of exploring the solution space, which contributes to finding the solutions in the upper-right corner. Since the only difference between the solution $\left(\mathrm{C}_{2}\right)$ evolved by the method 3 and the solution evolved by the method $2 \mathrm{D}$ is the weight factor, i.e., the weight factors evolved by the methods 3 and $2 \mathrm{D}$ are 0.58 and 0.7 , respectively, this gives us the conjecture that the weight factors from 0.58 to 0.7 derives the good solutions. However, the solutions with the 0.6 weight factor are worse than that with the 0.7 weight factor from Figure 19. This fact suggests that the method 3 can find a very niche solution by finding the 0.58 weight factor. 


\section{- Exploration of high profit solutions}

Focusing on the on-peak month (i.e., the vertical axis) in Figure 19, the method 3 can evolve the solution $\left(\mathrm{C}_{1}\right)$ that has higher profit than the solution $(\mathrm{B})$ evolved by PriGA as the conventional method. We compare these two solutions, $\left(\mathrm{C}_{1}\right)$ and $(\mathrm{B})$. Since two solutions are feasible, which means that the both flight networks can carry all passengers to their destination, the profit difference between two solutions come from the cost difference between of them.

Table 12 Comparison of flight routes in the proposed method and the conventional one

\begin{tabular}{lccc}
\hline \multirow{2}{*}{ Route number } & \multicolumn{3}{c}{ Conventional method solution (B) } \\
\cline { 2 - 4 } & Assigned order & Transportation cost (yen) & Fleet assignment \\
\hline Route 1 & 3 & $6,919,212$ & Large 2 \\
Route 2 & 2 & $23,165,390$ & Medium 1, large 11 \\
Route 3 & 7 & $8,473,266$ & Small 3, medium 3 \\
Total & & $38,557,868$ & Small 3, medium 4, large 13 \\
\hline \multirow{2}{*}{ Route number } & \multicolumn{3}{c}{ Conventional method solution $\left(C_{1}\right)$} \\
\cline { 2 - 4 } & Assigned order & Transportation cost (yen) & Fleet assignment \\
\hline Route 1 & 2 & $7,519,044$ & Small 1, medium 1, large 1 \\
Route 2 & 4 & $25,152,352$ & Small 2, medium 2, large 10 \\
Route 3 & 7 & $5,737,776$ & Medium 1, large 2 \\
& & $38,409,172$ & Small 3, medium 4, large 13 \\
\hline
\end{tabular}

From this fact, we analyse the cost difference between two solutions $\left(\mathrm{C}_{1}\right)$ and $(\mathrm{B})$ as shown in Table 12. Note that this table only shows the flight routes that have the different fleet assignment in the solution $(B)$ and $\left(\mathrm{C}_{1}\right)$. In detail, the route number, the assigned order, the transportation cost, the fleet assignment indicate the label of the route, the assigned order of fleets, the transportation cost of the route, and the type and number of the assigned fleets, respectively. Since the large type fleets (e.g., B777) does not require a lot of cost (i.e., the low cost) in comparison with the middle and small type fleets (e.g., B767), it is important to appropriately assign the large type fleets among three routes.

Since the solution $\left(C_{1}\right)$ evolved by the method 3 assigns the regular flights slightly fewer by discounting the passengers' demand according to the weight factor, the large type fleets remain until assigning the fleets in the last route. This contributes to appropriately assigning the large type fleets among three routes. Since the solution (B) evolved by PriGA as the conventional method, on the other hand, assigns the regular flights without discounting the passengers' demand, the large type fleets are firstly assigned in the route with a high assigned order [all large type fleets are assigned in the only routes 2 and 3 in the solution (B)]. This increases the total transportation cost. This is main reason why the method 3 can find the solution $\left(C_{1}\right)$ that has higher profit than the solution (B).

\section{- Flight network affected by double self-adaptive methods}

Figure 20 shows the flight network optimised by the method 3 that has the highest sum profit of the on- and off-peak months [i.e., the solution $\left(C_{1}\right)$ in Figure 19(b)]. In these figures, the left figure indicates the regular flight network, while the upper-right and 
lower-right figures indicate the non-regular one of the off- and on-peak months, respectively. The nodes and the arrows indicate the airports and the flight route, respectively.

Specifically, comparing the flight network optimised by the method 3 (shown in Figure 20) and that by the method 2D [shown in Figure 19(b)], the total number of the large airplanes is 36 in the regular flight in the case of the method 3, while that is 13 in the case of the method 2D. Since the large type fleets (e.g., B777) does not require a lot of cost (i.e., the low cost) as described in the previous discussion, the method 3 can reduce the cost by increasing an assignment of the large type fleets in the regular flight in comparison with the method 2D.

The non-regular flight rate in the whole flights in the method 3 is approximately $18.5 \%$. This rate is calculated by the average of non-regular flights between on- and off-peak month (10)/(the regular flight (44) + the average of non-regular flights (10)), where the average of non-regular flights is calculated as $10(=(5$ (3 large +2 small airplanes in off-peak month) +15 (4 large +6 medium +5 small airplanes in on-peak month))/2), and the regular flight is calculated as 44 (36 large +8 medium airplanes). Since additional efforts of generating the non-regular flights are needed as the non-regular flight rate increases, it is very important for airline companies to reduce the non-regular flight rate. Comparing the current non-regular flights rate [approximately $30 \%$ (Sato et al., 2001)], the rate of the proposed method is very smaller than the current one.

Figure 20 Flight network optimised by method 3 (see online version for colours)

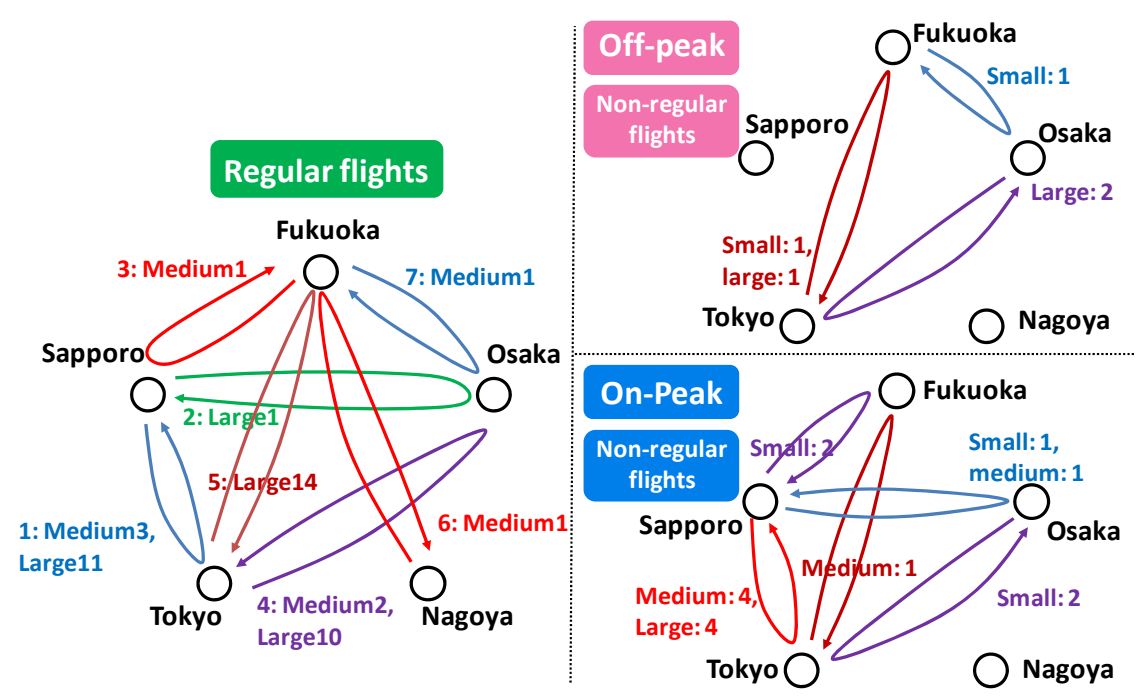

The above features of the proposed method are very strong advantage of the double self-adaptive fleet assignment method.

- $\quad$ Comparison with the three proposed methods

From the results of the experiments 1, 2, and 3, the comparison with three proposed methods [i.e., the MIN/MAX-based fleet assignment method (as the method 1), the self-adaptive MIN/MAX-based and deterministic weight-based fleet assignment method 
(as the method 2), and the double self-adaptive fleet assignment method (as the method 3)] are summarised as follows:

1 the solutions evolved by the method 3 are better than the solutions evolved by the method 2 (see Section 5.4.2), and the solutions evolved by the method 2 include the solutions evolved by the method 1 (see Section 5.3.2), which means that the method 3 is the best among three proposed methods

2 the good solutions can be evolved by the method 3 because of:

a a niche solution exploration by a double self-adaptive turning parameters

$\mathrm{b}$ an assignment of the low cost fleets to all routes as much as possible

c the small rate of non-regular flights in the whole flights (see Section 5.4.3).

Since the FAP in this paper requires the appropriate balance between the regular and non-regular flights to find good solutions, a niche solution exploration by a double self-adaptive turning parameters approach contributes to assigning the low cost fleets to all routes appropriately with not simply increasing the rate of non-regular flights (if the balance between the regular and non-regular flights is fixed, an appropriate assignment of the low cost fleets is hard to be achieved). This is the main reason why the proposed method 3 can solve the FAP appropriately.

\section{Conclusions}

This paper proposed the new multi-objective fleet assignment method for the flight network optimisation that assigns aircrafts for both the regular and non-regular flights. Concretely, we proposed:

a the MIN/MAX-based fleet assignment method (as the method 1)

b the self-adaptive MIN/MAX-based and deterministic weight-based fleet assignment method (as the method 2)

c the double self-adaptive fleet assignment method (as the method 3).

To investigate the effectiveness of our method, this paper applied them to Japanese domestic airport network optimisation for two months, on- and off-peak months, using a real-world data. The intensive experiments have revealed the following implications:

1 all three proposed methods can evolve a flight network of five airports that can be applied into the on- and off-peak month

2 the methods 2 and 3 can find the flight network that has a well-balanced profit between on- and off-peak months, i.e., the proposed method can find the flight network that has a potential of earning approximately 992 and 700 million yen in the on-peak and off-peak months which is the mostly same profit of actual ones even though the non-regular flight rate in the whole flights is approximately $18.5 \%$ which is less than the actual rate of $30 \%$ 
3 in peak month, the method 3 can find a flight network that has higher profit (approximately 0.2 million yen) than the conventional method even though the method 3 has to consider two months while the conventional method only considers a single month in the flight network optimisation.

What should be noticed here is that these results have only been obtained from the specific case, i.e., the flight network with five airports. Therefore, further careful qualifications and justifications, such as an increase of the airports, are needed to generalise our results. Such a scalability issue is important, but the flight network of the major routes can be optimised by the proposed method because the major routes are covered by the small number of airports (e.g., five airports in All Nippon Airways and seven airports in United Airline, i.e., San Francisco, Los Angeles, Denver, Huston, Chicago, Washington, and New York). Since the most of the profit of airline companies is earned in the major routes, the flight network optimisation of the major route is the primary concern of airline companies. From this viewpoint, the results provided by the proposed method in this research are sufficient even in the small number of airports. However, the scalability issue must be done in near future as the general perspective. In addition to this direction, the following future research must be pursued:

1 a consideration of other costs besides the transportation cost

2 a flight network optimisation by considering the regular flight rate

3 a flight schedule optimisation by considering a departure time.

\section{References}

Abara, J. (1989) 'Applying integer linear programming to the fleet assignment problem', Interfaces, Vol. 19, No. 4, pp.20-28.

Adachi, N., Sato, M. and Kobayashi, S. (2004) 'Application of genetic algorithm to flight schedule planning', System and Computers in Japan, Vol. 35, No. 12, pp.83-92.

ANA Japan [online] http://www.ana.co.jp/ (accessed 2015.9.10).

Barnhart, C., Boland, N.L., Clarke, L.W. and Shenoi, R.G. (1998) 'Flight string models for aircraft fleeting and routing', Transportation Science, Vol. 32, No. 3, pp.208-22.

Clarke, L., Johnson, E.L., Nemhauser, G.L. and Zhu, Z. (1997) 'The aircraft rotation problem', Annals of Operations Research, Vol. 69, pp.33-46.

Coello, C.A.C., Lamont, G.B. and Veldhuizen, D.A.V. (2007) Evolutionary Algorithms for Solving Multi-Objective Problems, 2nd ed., Springer, New York.

Dantzig, G.B. and Ramser, J.H. (1959) 'The truck dispatching problem', Management Science, Vol. 6, No. 1, pp.80-91.

Deb, K. (2001) Multi-Objective Optimization Using Evolutionary Algorithms, John Wiley \& Sons, New York.

Deb, K., Pratap, A., Agarwal, S. and Meyarivan, T. (2002) 'A fast and elitist multiobjective genetic algorithm: NSGA-II, IEEE Transactions on Evolutionary Computation, Vol. 6, No. 2 , pp.182-197.

Gen, M., Cheng, R. and Lin, L. (2008) Network Models and Optimization: Multi-Objective Genetic Algorithm Approach, Springer, London.

Gopalan, R. and Talluri, K.T. (1998) 'The aircraft maintenance routing problem', Operations Research, Vol. 46, No. 2, pp.260-271. 
Gu, Z., Johnson, E.L., Nemhauser, G.L. and Wang, Y. (1994) 'Some properties of the fleet assignment problem', Operations Research Letters, Vol. 15, No. 2, pp.59-71.

Hane, C.A., Barnhart, C., Johnson, E.L., Marsten, R.E., Nemhauser, G.L. and Sigismondi, G. (1995) 'The fleet assignment problem: solving a large-scale integer program', Mathematical Programming, Vol. 70, No. 2, pp.211-232.

Inoue, H. (2012) 'Simulation analysis of airline network with various aircraft', Journal of Economics, Vol. 18, Nos. 3/4, pp.53-73, Kumamoto Gakuen University.

Lobo, F.J., Lima, C.F. and Michalewicz, Z. (2007) 'Parameter setting in evolutionary algorithms', in Studies in Computational Intelligence, Springer-Verlag, Berlin Heidelberg.

Sato, M. and Adachi, N. (2001) 'Airline schedule planning by genetic algorithms', The Japanese Society for Artificial Intelligence, Vol. 16, No. 6, pp.493-500 (In Japanese).

Sato, M., Matumoto, S., Teramoto, Y. and Adachi, N. (2001) 'The fully dated airline crew pairing by genetic algorithms', The Japanese Society for Artificial Intelligence, Vol. 16, No. 3, pp.324-332 (In Japanese). 